\title{
Colorectal neoplasia differentially expressed: a long noncoding RNA with an imperative role in cancer
}

This article was published in the following Dove Press journal:

OncoTargets and Therapy

\section{Meiyu Dai \\ Shan Li \\ Xue Qin}

Department of Clinical Laboratory, First Affiliated Hospital of Guangxi Medical University, Nanning 53002I, Guangxi, China
Correspondence: Xue Qin Department of Clinical Laboratory, First Affiliated Hospital of Guangxi Medical University, No 6, Shuangyong Road, Nanning, Guangxi 53002I, China Tel +86 77| 5356052

Fax +86 77I 865353342

Email qinxue919@।26.com

\begin{abstract}
Colorectal neoplasia differentially expressed (CRNDE), as a long noncoding RNA (lncRNA), has attracted increasing attention in recent years and has been documented to be at abnormally high expression in various types of cancer, such as colorectal cancer, glioma, hepatocellular carcinoma, lung cancer, and breast cancer. It could not only be used as a clinical biomarker for the early diagnosis and prognosis evaluation in a variety of cancers but also promote the development and progress of various tumor cells. Moreover, it is involved in the targeting regulation of multiple microRNAs and the activation/inhibition of multiple signaling pathways. In this review, we presented a systematic summary of the potential carcinogenicity and clinical value of CRNDE in the current evidence, so as to provide reference for early diagnosis, prognosis evaluation, and targeted therapy of various clinical cancers.
\end{abstract}

Keywords: long noncoding RNA, CRNDE, cancer, biological function

\section{Introduction}

Long noncoding RNA (lncRNA) is a class of RNA molecules with a transcription length of $>200$ nt. ${ }^{1,2}$ They do not encode proteins but regulate the expression levels of genes in a variety of ways, such as epigenetic regulation, transcriptional regulation, and post-transcriptional regulation. IncRNA is characterized by its poor sequence conservation, low expression level, environmental susceptibility, and complex and diverse mode of action. In recent years, much progress has been made in the study of the relationship between lncRNA and diseases, especially in cancers. Researchers have found an important mechanism by which lncRNA metastasis-associated lung adenocarcinoma transcript 1 (MALAT1) promotes the metastasis of lung cancer. ${ }^{3}$ By interfering with MALAT1, they are expected to develop a method of inhibiting the metastasis of lung cancer. At the same time, there is a class of tumor suppressor lncRNA that is different from oncogenic one, such as maternally expressed gene 3 (MEG3). ${ }^{4}$ MEG3 is the first lncRNA to be found as a tumor suppressor. At present, the function of lncRNA needs to be further studied. Various lncRNA databases are constantly created and updated. Some new mechanisms, such as competing endogenous RNA (ceRNA) and microRNA (miRNA) sponges, are also being deployed around lncRNA. ${ }^{5}$ Thus, the research in this field of lncRNA presents a scene in full swing.

According to genomic location, IncRNAs are mainly divided into stand-alone lncRNAs, natural antisense transcripts, pseudogenes, IncRNAs, divergent transcripts, promoterassociated transcripts, and enhancer RNAs. ${ }^{6}$ This article focuses on Long intergenic 
noncoding (linc) RNA colorectal neoplasia differentially expressed (CRNDE), which also has many other gene synonyms, such as CRNDEP, PNAS-108, lincIRX5, LINC00180, and NCRNA00180. In 2011, it was officially named CRNDE. ${ }^{7}$ CRNDE is originally found to be significantly upregulated in colorectal cancer (CRC) tissues by database-mining analysis. ${ }^{8}$ The coding gene is localized in Chr16:hCG_1815491, and 14 different transcripts have been identified at present. Recent studies indicate that CRNDE is not only differentially expressed in CRC tissues but also abnormally expressed in various cancers, including lung cancer, ${ }^{9}$ hepatocellular carcinoma (HCC), ${ }^{10}$ renal cell carcinoma (RCC), ${ }^{11}$ ovarian cancer (OC), ${ }^{12}$ and glioma. ${ }^{13}$ At the same time, CRNDE is also closely related to disease diagnosis, ${ }^{14}$ prognosis, ${ }^{12}$ and some important biological functions ${ }^{15,16}$ in a variety of cancers. The purpose of this article is to comprehensively analyze the related research progress of CRNDE and to investigate its differential expression in tumor, biological regulation mechanism, and potential clinical values. It can provide an important reference for the diagnosis, prognosis evaluation, and targeted therapy of CRNDE in cancers.

\section{Biological structure of CRNDE}

CRNDE contains a total of six exons, five of which are core exons (E1, E2, and E4-E6) and one of which is less mentioned exon (E3), which is located on chromosome 16: 54,845,189-54,929,189 reverse strand next to IRX5 gene (Figure 1). Till now, the CRNDE gene encodes 14 splice variants, which are named as CRNDE-201-CRNDE-214 and can be found in the Ensembl database (Human GRCh38.p10) (Figure 2). Because of its complex splicing results, the exons often appear in different combinations, while the number of introns retained remains different. ${ }^{7}$ Different splicing patterns can cause RNA transcripts to vary in length. Alternative splicing is the key mechanism for the generation of protein diversity. In addition, the alternative splicing variants of a protein can exhibit different biological functions, or even opposite functions. ${ }^{17,18}$ Universal splicing disorder was a common feature of cancer cells, and some specific alternative splicing has been shown to be related to disease, ${ }^{19}$ especially in the pathogenesis of CRC. ${ }^{20}$

\section{Biological functions of CRNDE in a variety of cancers}

At present, the expression of CRNDE is abnormal in various cancers, which has been identified by many scholars. Therefore, further research should be focused on the biological functions and molecular mechanisms of CRNDE in various malignancies. As shown in the following passage, we presented a systematic summary of current research on the biomarker functions, mechanisms, and signaling pathways of CRNDE in malignancies.

\section{CRC}

CRNDE first showed the high expression level in neoplastic colorectal tissues through Affymetrix HG U133 array data. The results indicated that CRNDE showed significant high expression in $>90 \%$ of adenoma and adenocarcinoma tissues relative to normal tissues. This discovery was quickly confirmed by the method of microarray technology and quantitative real-time polymerase chain reaction (qRT-PCR). At the same time, the expression level of CRNDE in cell line and

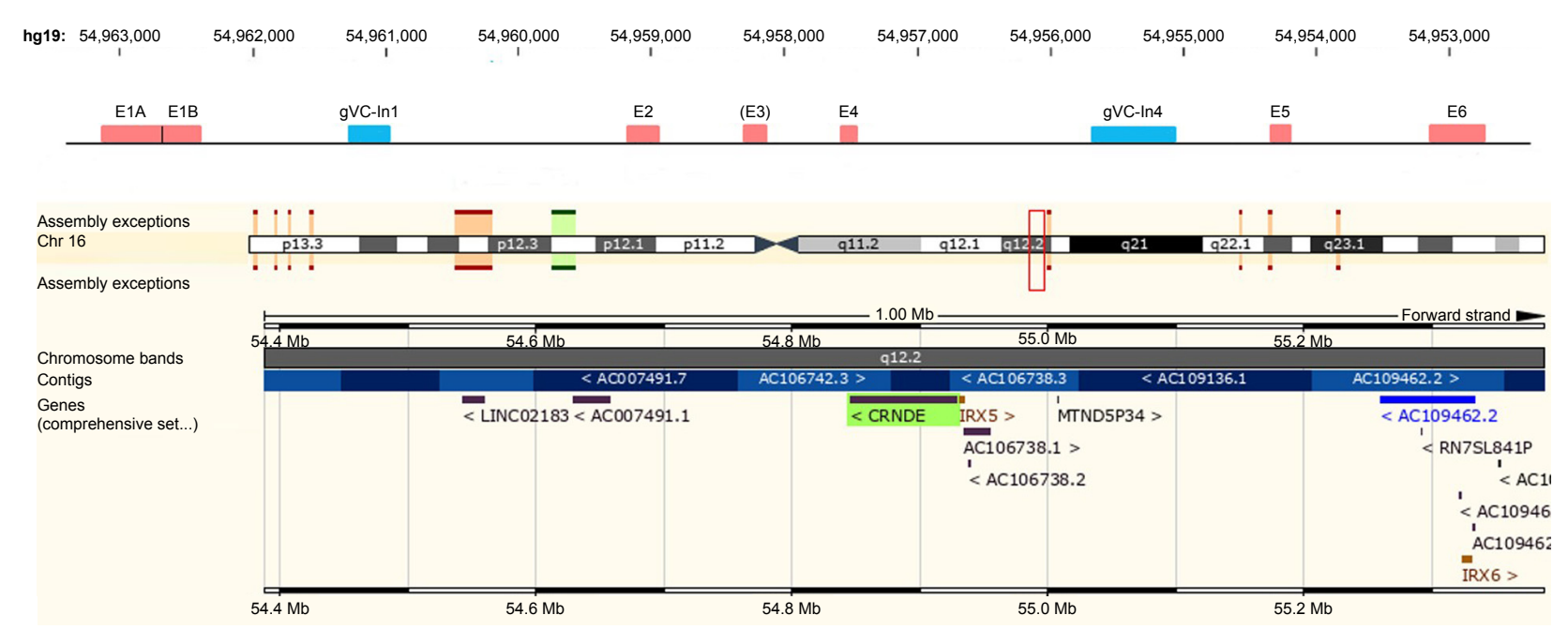

Figure I Genomic structure of CRNDE was captured from Ensemble genome browser. ${ }^{73}$

Abbreviation: CRNDE, colorectal neoplasia differentially expressed. 


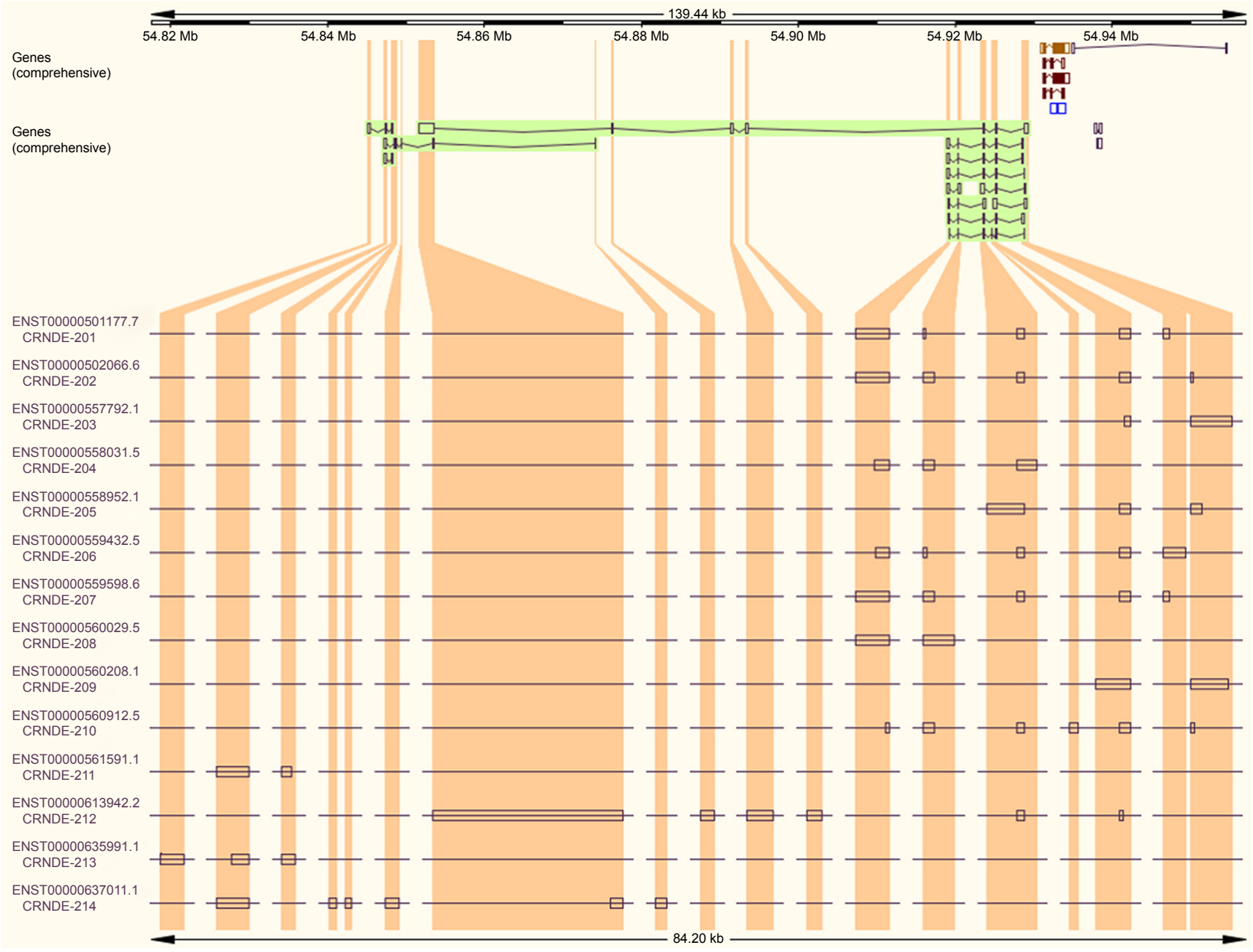

Figure 2 Schematic diagram of different transcripts of CRNDE was captured from Ensemble genome browser. ${ }^{73}$

Abbreviation: CRNDE, colorectal neoplasia differentially expressed.

plasma was validated. The results showed that CRNDE was highly expressed in the tissues, cells, and plasma of patients with CRC. ${ }^{7}$ Our team also studied the expression levels of CRNDE in many aspects, such as CRC tissues, cell lines, and serum. The results further confirmed that CRNDE was highly expressed in the tissues, serum, and cell lines of patients with CRC. ${ }^{21}$ Moreover, CRNDE was abnormally expressed in other tissues, serum, and cell lines of various malignant tumors. The detailed information is listed in Table 1. With the improvement in living standards and the changes in diet structures, the incidence of CRC is increasing year by year. ${ }^{22}$ Now, CRC is the third most common cancer, which ranks fourth among cancer-related deaths worldwide. ${ }^{23}$ Therefore, the early diagnosis, prognosis evaluation, target therapy, and the study of the mechanisms of tumor development are especially important for patients with CRC. Proteins, ${ }^{24}$ lipids, ${ }^{25}$ and genes (especially miRNA, lncRNA, and circRNA) ${ }^{26-28}$ are the focuses of research for clinical biomarkers. Serological biomarkers are more common in early diagnosis because of their convenient sampling and minimally invasive. The studies of the expression level of CRNDE in serum/plasma are currently directed at patients with CRC. ${ }^{7,21}$ At the same time, some scholars extracted the exosomes from the serum of patients with CRC and detected the expression of CRNDE accurately. The results showed that CRNDE was highly expressed in CRC samples and could further differentiate patients with colorectal benign diseases from healthy subjects with high sensitivity and specificity, and the high exosomal CRNDE expression level predicted poor overall survival rate. ${ }^{14} \mathrm{~A}$ recent study showed that serum exosomal CRNDE-p combined with miRNA-217 could improve the diagnostic efficacy of CRC..$^{29}$ CRNDE-p is a subtype of a number of transcripts in CRNDE. Moreover, CRNDE overexpression as an indicator of prognostic evaluation in tissues with abnormal expression had significant clinical value in CRC. In addition, CRNDE, as an indicator of tumor prognosis, 
Table I CRNDE expression in various malignant tumors

\begin{tabular}{|c|c|c|c|c|}
\hline Cancer type & Sample & Method & Expression & References \\
\hline CRC & Tissue, serum, cell, exosome & GEO, ${ }^{a}$ qRT-PCR, microarray & Upregulated & $6,7,14,21,29-34$ \\
\hline PTC & Tissue & TCGA, ${ }^{a}$ GEO ${ }^{a}$ & Upregulated & 59,64 \\
\hline LAD & Tissue, cell & qRT-PCR & Upregulated & 9 \\
\hline Glioma & Tissue, cell & GEO, ${ }^{a}$ qRT-PCR, microarray & Upregulated & $13,15,16,38,65,66$ \\
\hline GC & Tissue, cell & qRT-PCR & Upregulated & $39,60,67$ \\
\hline NSCLC & Tissue, cell & qRT-PCR & Upregulated & 53 \\
\hline BC & Tissue, cell & qRT-PCR & Upregulated & 56 \\
\hline MM & Tissue, cell & qRT-PCR & Upregulated & 61 \\
\hline $\mathrm{HCC}$ & Tissue, cell, serum & qRT-PCR, RNA-seq & Upregulated & $10,49,50,68$ \\
\hline $\mathrm{RCC}$ & Tissue, cell & Oncomine, ${ }^{\mathrm{a}} \mathrm{qRT}-\mathrm{PCR}$ & Upregulated & 11,69 \\
\hline Medulloblastoma & Tissue, cell & qRT-PCR & Upregulated & 41 \\
\hline $\mathrm{CC}$ & Tissue, cell & qRT-PCR & Upregulated & 70 \\
\hline Osteosarcoma & Tissue, cell & qRT-PCR & Upregulated & 71 \\
\hline PC & Tissue, cell & qRT-PCR & Upregulated & 72 \\
\hline
\end{tabular}

Note: ${ }^{a}$ Differentially expressed CRNDE was obtained by mining the corresponding database.

Abbreviations: BC, breast cancer; CC, cervical cancer; CRC, colorectal cancer; CRNDE, colorectal neoplasia differentially expressed; GC, gastric cancer; GEO, Gene Expression Omnibus; HCC, hepatocellular carcinoma; LAD, lung adenocarcinoma; MM, multiple myeloma; NSCLC, non-small-cell lung carcinomas; PC, pancreatic cancer; PTC, papillary thyroid cancer; qRT-PCR, quantitative real-time polymerase chain reaction; RCC, renal cell carcinoma; TCGA, The Cancer Genome Atlas.

has been confirmed by a number of studies that CRNDE was highly expressed in CRC tissues, suggesting a poor outcome. ${ }^{30-32}$ In addition to the study of clinical biomarker, the mechanisms of CRNDE in CRC have been intensively studied by many scholars. The upregulated CRNDE could accelerate CRC cell proliferation and migration by activating Ras/mitogen-activated protein kinase (MAPK) signaling pathway. Meanwhile, the stability of CRNDE was influenced by heterogeneous nuclear ribonucleoprotein U-like 2 protein (hnRNPUL2), which was accumulated in the cytoplasm. ${ }^{30}$ Moreover, the low expression of CRNDE could inhibit the cell progression by competitively binding miRNA-217 to increase the expression of TCF7L2 and the activity of the Wnt/ $\beta$-catenin signaling pathway. ${ }^{33}$ CRNDE knockdown has also been shown to increase the expression of miRNA$181 \mathrm{a}-5 \mathrm{p}$, thereby inhibiting cell proliferation and reducing chemical resistance by inhibiting the Wnt/ $\beta$-catenin signaling pathway. ${ }^{31}$ Thus, Wnt/ $\beta$-catenin signaling pathway was a key mechanism for CRNDE to regulate the development and progression of CRC. With respect to drug resistance, a related article has reported that high expression of CRNDE with oxaliplatin treatment could reduce DNA damage and cell apoptosis. Further research on the mechanism of this phenomenon showed that CRNDE served as a ceRNA to enhance oxaliplatin resistance by sponging miRNA-136 in the CRC cells. ${ }^{34}$ In conclusion, CRNDE could activate or inhibit the Ras/MAPK and Wnt/ $\beta$-catenin signaling pathways by competitively binding miRNAs, thereby further regulating CRC cell proliferation, invasion, apoptosis, metastasis, and chemical resistance.

\section{Glioma}

Glioma is the most common primary brain tumor arising from glial cell carcinogenesis in the brain and spinal cord and has very high recurrence rates and mortality rates. ${ }^{35}$ Glioblastoma multiforme (GBM), in particular, is the most aggressive malignancy in gliomas, and the average survival time is $<15$ months after standardized treatment. ${ }^{36,37}$ CRNDE not only played an important role in $\mathrm{CRC}$ but also was closely related to the clinical and functional studies of gliomas. As mentioned earlier, CRNDE was highly expressed in glioma tissues compared with normal brain tissues. ${ }^{37}$ Survival analysis showed that the high expression of CRNDE was negatively correlated with the prognosis and overall survival of the patients with glioma. ${ }^{13,38}$ Multivariate Cox regression analysis showed that CRNDE could be used as an independent risk factor to evaluate the prognosis of patients with glioma. ${ }^{13}$ In addition, CRNDE involves multiple classic signaling pathways in the development of glioma, including TLR3-NF- $\mathrm{KB}-$ cytokine signaling pathway, epidermal growth factor receptor (EGFR) signaling pathway, and mammalian target of rapamycin (mTOR) signaling pathway. Li et $\mathrm{al}^{39}$ speculated that CRNDE triggered inflammation through the TLR3-NF- $\mathrm{B}$-cytokine signaling pathway, further regulating the occurrence and development of tumors. Kiang et $\mathrm{al}^{38}$ also found that EGF and EGFR tyrosine kinase inhibitors could regulate the expression of CRNDE, which further affected the growth of glioma cells. This finding implied that CRNDE might be the downstream activator of EGFR. Moreover, related mechanism studies further showed that the promoter region of histone acetylation might lead 
to CRNDE overexpression through the mTOR signaling pathway. ${ }^{16}$ The results provided important reference for the study of targeted therapy of glioma. In addition to these classical pathways, some scholars discovered that CRNDE could regulate the cell malignant behavior by disturbing miR-384/ PIWIL4/STAT3 axis, which might be a novel pathway for targeted research of glioma. ${ }^{40}$ There were also reports of studies on glioma stem cells (GSC). CRNDE overexpression could reduce the expression of miRNA-186 in GSC, thereby further inhibiting the apoptosis and promoting the proliferation, invasion, and metastasis of GSC. ${ }^{15}$ High expression of CRNDE also promoted medulloblastoma cell growth. ${ }^{41}$ All these manifested that CRNDE was particularly important in the research of brain cancer.

\section{HCC}

$\mathrm{HCC}$ is one of the most common malignant tumors in the world, which originates from hepatocytes or intrahepatic bile duct cells. ${ }^{42} \mathrm{HCC}$ has a high incidence and is the second most common malignant tumor in China. ${ }^{43}$ Most patients diagnosed with $\mathrm{HCC}$ are in the middle and late stages and are often with poor prognosis. Many scholars were trying to find the satisfactory biomarkers for the early diagnosis and prognostic evaluation of HCC. ${ }^{44-46}$ There have been a lot of reports about the correlation between lncRNA and $\mathrm{HCC},{ }^{47,48}$ and the role of CRNDE for HCC was also very important. High-throughput sequencing and bioinformatics are the popular methods of detection and analysis at present. Esposti et $\mathrm{al}^{10}$ formally used this advanced method to screen out a series of abnormal expression of lncRNAs (included CRNDE) in HCC tissues compared with adjacent nontumor tissues and carried out experimental validation and related functional prediction analysis. At the same time, it has been reported that CRNDE was highly expressed in HCC tissues, which provided important value for the clinical diagnosis of HCC. Moreover, CRNDE overexpression could promote the proliferation, invasion, and migration of HCC cells. ${ }^{49}$ In this study, they also found that CRNDE could negatively regulate miRNA-384, thus affecting the expression of important genes NF- $\mathrm{KB}$ and $\mathrm{p}-\mathrm{AKT}$. Recently, the regulatory mechanisms and related signaling pathways of CRNDE in HCC have also been investigated. Through the gene knockout of CRNDE, they found that CRNDE could act on HCC cells by regulating the mTOR signaling pathway, so that it could play an important role in the antitumor proliferation and antitumor angiogenesis. ${ }^{50}$ These studies also provided important reference for the early diagnosis and gene targeted therapy of HCC.

\section{Lung cancer}

Lung cancer is the most common primary malignant tumor of the lung. The vast majority of lung cancer originates in the bronchial epithelium, so it is also called bronchogenic carcinoma. The incidence and mortality of lung cancer are very high and rise year by year, especially in China. ${ }^{51,52}$ In general, lung cancer can be divided into the following two major types: small cell lung cancer and non-small-cell lung cancer (NSCLC). NSCLC accounts for $\sim 80 \%$ of the total lung cancer, including lung adenocarcinoma (LAD), squamous cell carcinoma, and large cell lung cancer. Therefore, more emphasis was placed on the type of NSCLC in the study of lung cancer.

Liu et al were the first to study the role and possible mechanism of CRNDE in the development of NSCLC. They found that high expression of CRNDE promoted the proliferation and growth of NSCLC cells and also found that CRNDE could affect the expression levels of CDK4, CDK6, and CCNE1, thus activating the PI3K/AKT signaling pathway. ${ }^{53}$ In addition, some researchers have studied the role of CRNDE in LAD, which was the predominant subtype of NSCLC. The result indicated that high CRNDE expression in LAD patients showed a shorter overall survival and was closely related to the clinicopathological features of the patients, such as low differentiation, TNM stage, radiotherapy response, and lymph node metastasis. ${ }^{9}$ In addition, they found through rescue experiments that CRNDE could affect radio-sensitivity of LAD cells by interacting with PRC2 to regulate target gene $\mathrm{p} 21$. This discovery had important implications for the study of the radio-resistant phenotype of LAD.

\section{Breast cancer (BC)}

$\mathrm{BC}$ is one of the most common malignant tumors in women, mainly occurring in the mammary gland epithelial tissue. Approximately $99 \%$ of BC occurs in women, and its incidence is often associated with heredity. ${ }^{54,55}$ The study of the role of CRNDE in $\mathrm{BC}$ was similar to the cancers mentioned earlier. High expression of CRNDE in BC suggested poor prognosis and was closely related to some clinical features. Meanwhile, CRNDE overexpression could promote the proliferation, invasion, and metastasis of BC cells. ${ }^{56} \mathrm{~A}$ further study on its possible regulatory mechanisms have revealed that CRNDE could directly bind to miRNA-136 to activate the Wnt/ $\beta$-catenin signaling pathway. ${ }^{56}$ In addition, it has been reported that SF3B1 mutation could cause aberrant expression of multiple genes, including CRNDE, which might serve as a new target for $\mathrm{BC}$ treatment. ${ }^{57}$ Similarly, 
the effect of SF3B1 mutation on uveal melanoma has been studied in the same way. ${ }^{58}$

\section{Other cancers}

In addition to the tumors discussed earlier, there were some reports showing that CRNDE played an important role in some other cancers. In OC, CRNDE was a potentially important prognostic biomarker and the high expression of CRNDE was significantly negatively associated with prognosis in women with OC. ${ }^{12}$ In papillary thyroid cancer (PTC), Luo et al screened the differentially expressed IncRNAs based on the PTC copy number data from The Cancer Genome Atlas (TCGA) database. The expression profiles in the Gene Expression Omnibus (GEO) database were then used to validate the results. Finally, three lncRNAs were evaluated, including CRNDE. ${ }^{59}$ In the mechanism of mutual regulation with miRNA, they found that CRNDE could promote the proliferation and growth of GC cells and multiple myeloma cells by targeting the regulation of miRNA-145 and miRNA$451 .^{60,61}$ Study has also shown that CRNDE could act as a scaffold specifically to bind to genes DMBT1 and c-IAP1, thereby regulating the expression of tumor suppressor gene DMBT1 and activating the PI3K-AKT signaling pathway, which lead to the development of gallbladder carcinoma. ${ }^{62}$ Similar to the abovementioned types of cancers, high expression of CRNDE could also promote the cell proliferation of RCC by activating the Wnt/ $\beta$-catenin signaling pathway. ${ }^{11}$ In addition, CRNDE played an important role in nonsolid tumors and a study has shown that hypermethylation of CRNDE could contribute to the poor prognosis of chronic lymphocytic leukemia. ${ }^{63}$ This also provided new directions for multi-angle research of CRNDE.

\section{Conclusion and perspectives}

Ever since CRNDE was first named, it has been intensively studied in a variety of human cancers. So far, CRNDE as an oncogene has been shown to be highly expressed in all tumor tissues compared with the normal tissues in the published reports. As an emerging clinical biomarker, CRNDE could be used as an indicator of clinical diagnosis and prognosis for multiple cancers, especially in prognostic evaluation. As a clinical marker, there is still less research on patient

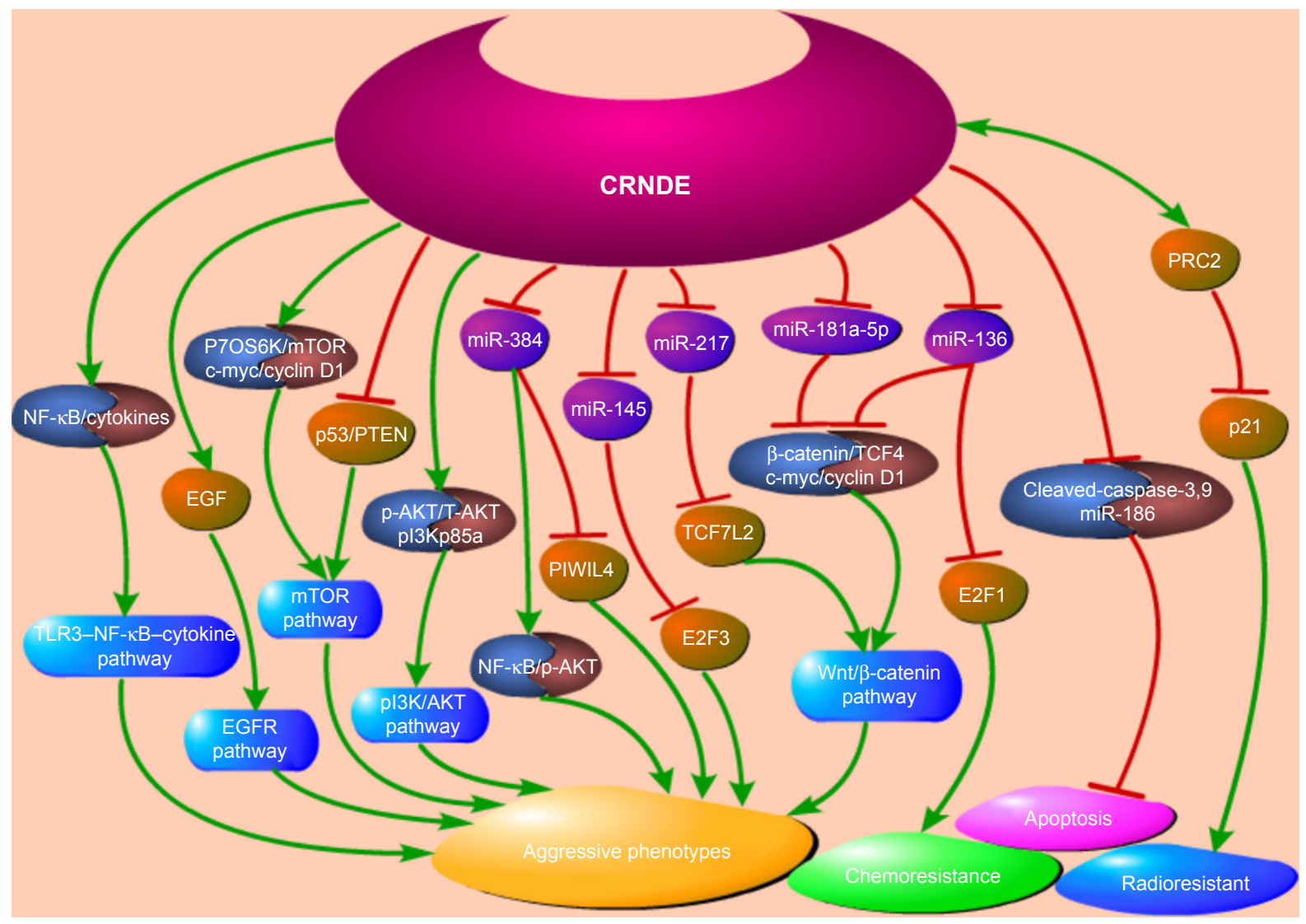

Figure 3 Molecular mechanism of CRNDE in cancer progression.

Note: The red lines indicate restraint, and the green lines indicate acceleration.

Abbreviations: CRNDE, colorectal neoplasia differentially expressed; EGFR, epidermal growth factor receptor; mTOR, mammalian target of rapamycin. 
serum, plasma, or other secretions, which needs to be further investigated. Meanwhile, more studies were aimed at the biological functions and related regulatory mechanisms and pathways of CRNDE in a variety of cancers. The high expression of CRNDE could promote the proliferation, invasion, or metastasis of tumor cells in most related cancers. In addition, CRNDE could be used as a miRNA sponge to competitively bind with specific miRNAs, thereby affecting the expression of related target genes and activating the associated signaling pathways. The main regulatory mechanisms are summarized in Figure 3. More research should be done about other cancers in the future, and the study model should be innovated since it is too traditional.

\section{Acknowledgment}

We owe our special thanks to Yujie Huang for her work in language editing.

\section{Author contributions}

XQ participated in the overall design of this article as the corresponding author. MD participated in the writing of the article as the first author. SL participated in the review and revision of the article. All authors contributed toward data analysis, drafting and revising the paper and agree to be accountable for all aspects of the work.

\section{Disclosure}

The authors report no conflicts of interest in this work.

\section{References}

1. Mercer TR, Mattick JS. Structure and function of long noncoding RNAs in epigenetic regulation. Nat Struct Mol Biol. 2013;20(3): 300-307.

2. Derrien T, Johnson R, Bussotti G, et al. The GENCODE v7 catalog of human long noncoding RNAs: analysis of their gene structure, evolution, and expression. Genome Res. 2012;22(9):1775-1789.

3. Gutschner T, Hammerle M, Eissmann M, et al. The noncoding RNA MALAT1 is a critical regulator of the metastasis phenotype of lung cancer cells. Cancer Res. 2013;73(3):1180-1189.

4. Zhang X, Gejman R, Mahta A, et al. Maternally expressed gene 3, an imprinted noncoding RNA gene, is associated with meningioma pathogenesis and progression. Cancer Res. 2010;70(6):2350-2358.

5. Thomson DW, Dinger ME. Endogenous microRNA sponges: evidence and controversy. Nat Rev Genet. 2016;17(5):272-283.

6. Ding J, Li J, Wang H, et al. Long noncoding RNA CRNDE promotes colorectal cancer cell proliferation via epigenetically silencing DUSP5/ CDKN1A expression. Cell Death Dis. 2017;8(8):e2997.

7. Graham LD, Pedersen SK, Brown GS, et al. Colorectal neoplasia differentially expressed (CRNDE), a novel gene with elevated expression in colorectal adenomas and adenocarcinomas. Genes Cancer. 2011;2(8):829-840.

8. Nagaraj SH, Reverter A. A boolean-based systems biology approach to predict novel genes associated with cancer: application to colorectal cancer. BMC Syst Biol. 2011;5:35.
9. Zhang M, Gao C, Yang Y, et al. Long noncoding RNA CRNDE/PRC2 participated in the radiotherapy resistance of human lung adenocarcinoma through targeting p21 expression. Oncol Res. 2017 Epub ahead of print.

10. Esposti DD, Hernandez-Vargas H, Voegele C, et al. Identification of novel long non-coding RNAs deregulated in hepatocellular carcinoma using RNA-sequencing. Oncotarget. 2016;7(22):31862-31877.

11. Shao K, Shi T, Yang Y, Wang X, Xu D, Zhou P. Highly expressed lncRNA CRNDE promotes cell proliferation through $\mathrm{Wnt}$ /beta-catenin signaling in renal cell carcinoma. Tumour Biol. 2016 Epub ahead of print.

12. Szafron LM, Balcerak A, Grzybowska EA, et al. The putative oncogene, CRNDE, is a negative prognostic factor in ovarian cancer patients. Oncotarget. 2015;6(41):43897-43910.

13. Jing SY, Lu YY, Yang JK, Deng WY, Zhou Q, Jiao BH. Expression of long non-coding RNA CRNDE in glioma and its correlation with tumor progression and patient survival. Eur Rev Med Pharmacol Sci. 2016;20(19):3992-3996.

14. Liu T, Zhang X, Gao S, et al. Exosomal long noncoding RNA CRNDE-h as a novel serum-based biomarker for diagnosis and prognosis of colorectal cancer. Oncotarget. 2016;7(51):85551-85563.

15. Zheng J, Li XD, Wang P, et al. CRNDE affects the malignant biological characteristics of human glioma stem cells by negatively regulating miR-186. Oncotarget. 2015;6(28):25339-25355.

16. Wang Y, Wang Y, Li J, Zhang Y, Yin H, Han B. CRNDE, a longnoncoding RNA, promotes glioma cell growth and invasion through mTOR signaling. Cancer Lett. 2015;367(2):122-128.

17. Srebrow A, Kornblihtt AR. The connection between splicing and cancer. J Cell Sci. 2006;119(pt 13):2635-2641.

18. Thorsen K, Sorensen KD, Brems-Eskildsen AS, et al. Alternative splicing in colon, bladder, and prostate cancer identified by exon array analysis. Mol Cell Proteomics. 2008;7(7):1214-1224.

19. Chen GL, Ma Q, Goswami D, Shang J, Miller GM. Modulation of nuclear REST by alternative splicing: a potential therapeutic target for Huntington's disease. J Cell Mol Med. 2017;21(11):2974-2984.

20. Snezhkina AV, Krasnov GS, Zaretsky AR, et al. Differential expression of alternatively spliced transcripts related to energy metabolism in colorectal cancer. BMC Genomics. 2016;17(suppl 14):1011.

21. Dai M, Chen X, Mo S, et al. Meta-signature LncRNAs serve as novel biomarkers for colorectal cancer: integrated bioinformatics analysis, experimental validation and diagnostic evaluation. Sci Rep. 2017;7:46572.

22. Zullig LL, Smith VA, Jackson GL, et al. Colorectal cancer statistics from the veterans affairs central cancer registry. Clin Colorectal Cancer. 2016;15(4):e199-e204.

23. Chen W, Zheng R, Zeng H, Zhang S, He J. Annual report on status of cancer in China, 2011. Chin J Cancer Res. 2015;27(1):2-12.

24. Dai M, Chen X, Liu X, Peng Z, Meng J, Dai S. Diagnostic value of the combination of golgi protein 73 and alpha-fetoprotein in hepatocellular carcinoma: a meta-analysis. PLoS One. 2015;10(10):e0140067.

25. Chen X, Chen H, Dai M, et al. Plasma lipidomics profiling identified lipid biomarkers in distinguishing early-stage breast cancer from benign lesions. Oncotarget. 2016;7(24):36622-36631.

26. Dai S, Lu Y, Long Y, et al. Prognostic value of microRNAs in cervical carcinoma: a systematic review and meta-analysis. Oncotarget. 2016; 7(23):35369-35378.

27. He B, Zeng J, Chao W, et al. Serum long non-coding RNAs MALAT1, AFAP1-AS1 and AL359062 as diagnostic and prognostic biomarkers for nasopharyngeal carcinoma. Oncotarget. 2017;8(25):41166-41177.

28. Zhu X, Wang X, Wei S, et al. hsa_circ_0013958: a circular RNA and potential novel biomarker for lung adenocarcinoma. FEBS J. 2017; 284(14):2170-2182.

29. Yu B, Du Q, Li H, et al. Diagnostic potential of serum exosomal colorectal neoplasia differentially expressed long non-coding RNA (CRNDE-p) and microRNA-217 expression in colorectal carcinoma. Oncotarget. 2017;8(48):83745-83753.

30. Jiang H, Wang Y, Ai M, et al. Long noncoding RNA CRNDE stabilized by hnRNPUL2 accelerates cell proliferation and migration in colorectal carcinoma via activating Ras/MAPK signaling pathways. Cell Death Dis. 2017;8(6):e2862. 
31. Han P, Li JW, Zhang BM, et al. The lncRNA CRNDE promotes colorectal cancer cell proliferation and chemoresistance via miR-181a$5 \mathrm{p}$-mediated regulation of Wnt/beta-catenin signaling. Mol Cancer. 2017; 16(1):9.

32. Liu T, Zhang X, Yang YM, Du LT, Wang CX. Increased expression of the long noncoding RNA CRNDE-h indicates a poor prognosis in colorectal cancer, and is positively correlated with IRX 5 mRNA expression. Onco Targets Ther. 2016;9:1437-1448.

33. Yu B, Ye X, Du Q, Zhu B, Zhai Q, Li XX. The long non-coding RNA CRNDE promotes colorectal carcinoma progression by competitively binding miR-217 with TCF7L2 and enhancing the Wnt/beta-catenin signaling pathway. Cell Physiol Biochem. 2017;41(6):2489-2502.

34. Gao H, Song X, Kang T, et al. Long noncoding RNA CRNDE functions as a competing endogenous RNA to promote metastasis and oxaliplatin resistance by sponging miR-136 in colorectal cancer. Onco Targets Ther. 2017;10:205-216.

35. Kiang KM, Zhang XQ, Leung GK. Long non-coding RNAs: the key players in glioma pathogenesis. Cancers (Basel). 2015;7(3):1406-1424.

36. Tate MC, Aghi MK. Biology of angiogenesis and invasion in glioma. Neurotherapeutics. 2009;6(3):447-457.

37. Johnson DR, O'Neill BP. Glioblastoma survival in the United States before and during the temozolomide era. J Neurooncol. 2012;107(2):359-364.

38. Kiang KM, Zhang XQ, Zhang GP, et al. CRNDE expression positively correlates with EGFR activation and modulates glioma cell growth. Target Oncol. 2017;12(3):353-363.

39. Li H, Li Q, Guo T, He W, Dong C, Wang Y. LncRNA CRNDE triggers inflammation through the TLR3-NF-kappaB-cytokine signaling pathway. Tumour Biol. 2017;39(6):1010428317703821.

40. Zheng J, Liu X, Wang P, et al. CRNDE promotes malignant progression of glioma by attenuating miR-384/PIWIL4/STAT3 Axis. Mol Ther. 2016;24(7):1199-1215.

41. Song H, Han LM, Gao Q, Sun Y. Long non-coding RNA CRNDE promotes tumor growth in medulloblastoma. Eur Rev Med Pharmacol Sci. 2016;20(12):2588-2597.

42. Yang X, Xie X, Xiao YF, et al. The emergence of long non-coding RNAs in the tumorigenesis of hepatocellular carcinoma. Cancer Lett. 2015;360(2):119-124.

43. Chen JG, Zhang SW. Liver cancer epidemic in China: past, present and future. Semin Cancer Biol. 2011;21(1):59-69.

44. Zhou F, Shang W, Yu X, Tian J. Glypican-3: a promising biomarker for hepatocellular carcinoma diagnosis and treatment. Med Res Rev. 2017;38(2):741-767.

45. Fan SS, Liao CS, Cao YD, et al. A low serum Tat-interacting protein 30 level is a diagnostic and prognostic biomarker for hepatocellular carcinoma. Oncol Lett. 2017;13(6):4208-4214.

46. Juarez-Hernandez E, Motola-Kuba D, Chavez-Tapia NC, Uribe M, Barbero Becerra V. Biomarkers in hepatocellular carcinoma: an overview. Expert Rev Gastroenterol Hepatol. 2017;11(6):549-558.

47. Jiang R, Tang J, Chen Y, et al. The long noncoding RNA lnc-EGFR stimulates T-regulatory cells differentiation thus promoting hepatocellular carcinoma immune evasion. Nat Commun. 2017;8:15129.

48. Yang Y, Chen L, Gu J, et al. Recurrently deregulated lncRNAs in hepatocellular carcinoma. Nat Commun. 2017;8:14421.

49. Chen Z, Yu C, Zhan L, Pan Y, Chen L, Sun C. LncRNA CRNDE promotes hepatic carcinoma cell proliferation, migration and invasion by suppressing miR-384. Am J Cancer Res. 2016;6(10):2299-2309.

50. Dong R, Liu XQ, Zhang BB, Liu BH, Zheng S, Dong KR. Long non-coding RNA-CRNDE: a novel regulator of tumor growth and angiogenesis in hepatoblastoma. Oncotarget. 2017;8(26):42087-42097.

51. Chen W, Zheng R, Baade PD, et al. Cancer statistics in China, 2015. CA Cancer J Clin. 2016;66(2):115-132.

52. DeSantis CE, Siegel RL, Sauer AG, et al. Cancer statistics for African Americans, 2016: progress and opportunities in reducing racial disparities. CA Cancer J Clin. 2016;66(4):290-308.

53. Liu XX, Xiong HP, Huang JS, Qi K, Xu JJ. Highly expressed long non-coding RNA CRNDE promotes cell proliferation through PI3K/ AKT signalling in non-small cell lung carcinoma. Clin Exp Pharmacol Physiol. 2017;44(8):895-902.
54. Kenny T, Schmidt H, Adelson K, et al. Patient-derived interstitial fluids and predisposition to aggressive sporadic breast cancer through collagen remodeling and inactivation of p53. Clin Cancer Res. 2017; 23(18):5446-5459.

55. Sun J, Meng H, Yao L, et al. Germline mutations in cancer susceptibility genes in a large series of unselected breast cancer patients. Clin Cancer Res. 2017;23(20):6113-6119.

56. Huan J, Xing L, Lin Q, Xui H, Qin X. Long noncoding RNA CRNDE activates $\mathrm{Wnt} /$ beta-catenin signaling pathway through acting as a molecular sponge of microRNA-136 in human breast cancer. Am J Transl Res. 2017;9(4):1977-1989.

57. Maguire SL, Leonidou A, Wai P, et al. SF3B1 mutations constitute a novel therapeutic target in breast cancer. J Pathol. 2015;235(4):571-580.

58. Furney SJ, Pedersen M, Gentien D, et al. SF3B1 mutations are associated with alternative splicing in uveal melanoma. Cancer Discov. 2013;3(10):1122-1129.

59. Luo YH, Liang L, He RQ, et al. RNA-sequencing investigation identifies an effective risk score generated by three novel lncRNAs for the survival of papillary thyroid cancer patients. Oncotarget. 2017;8(43):74139-74158.

60. Hu CE, Du PZ, Zhang HD, Huang GJ. Long noncoding RNA CRNDE promotes proliferation of gastric cancer cells by targeting miR-145. Cell Physiol Biochem. 2017;42(1):13-21.

61. Meng YB, He X, Huang YF, Wu QN, Zhou YC, Hao DJ. Long noncoding RNA CRNDE promotes multiple myeloma cell growth by suppressing MiR-451. Oncol Res. 2017;25(7):1207-1214.

62. Shen S, Liu H, Wang Y, et al. Long non-coding RNA CRNDE promotes gallbladder carcinoma carcinogenesis and as a scaffold of DMBT1 and C-IAP1 complexes to activating PI3K-AKT pathway. Oncotarget. 2016; 7(45):72833-72844.

63. Subhash S, Andersson PO, Kosalai ST, Kanduri C, Kanduri M. Global DNA methylation profiling reveals new insights into epigenetically deregulated protein coding and long noncoding RNAs in CLL. Clin Epigenetics. 2016;8:106.

64. Sun H, He L, Ma L, et al. LncRNA CRNDE promotes cell proliferation, invasion and migration by competitively binding miR-384 in papillary thyroid cancer. Oncotarget. 2017;8(66):110552-110565.

65. Chen Y, Wu JJ, Lin XB, et al. Differential lncRNA expression profiles in recurrent gliomas compared with primary gliomas identified by microarray analysis. Int J Clin Exp Med. 2015;8(4):5033-5043.

66. Li DX, Fei XR, Dong YF, et al. The long non-coding RNA CRNDE acts as a ceRNA and promotes glioma malignancy by preventing miR136-5p-mediated downregulation of Bcl-2 and Wnt2. Oncotarget. 2017;8(50):88163-88178

67. Du DX, Lian DB, Amin BH, Yan W. Long non-coding RNA CRNDE is a novel tumor promoter by modulating PI3K/AKT signal pathways in human gastric cancer. Eur Rev Med Pharmacol Sci. 2017;21(23):5392-5398.

68. Dai M, Chen S, Wei X, et al. Diagnosis, prognosis and bioinformatics analysis of lncRNAs in hepatocellular carcinoma. Oncotarget. 2017; 8(56):95799-95809.

69. Yang FY, Wang Y, Wu JG, et al. Analysis of long non-coding RNA expression profiles in clear cell renal cell carcinoma. Oncol Lett. 2017; 14(3):2757-2764.

70. Meng Y, Li Q, Li L, Ma R. The long non-coding RNA CRNDE promotes cervical cancer cell growth and metastasis. Biol Chem. 2017; 399(1):93-100.

71. Li Z, Tang Y, Xing W, Dong W, Wang Z. LncRNA, CRNDE promotes osteosarcoma cell proliferation, invasion and migration by regulating Notch1 signaling and epithelial-mesenchymal transition. Exp Mol Pathol. 2017;104(1):19-25.

72. Wang G, Pan J, Zhang L, Wei Y, Wang C. Long non-coding RNA CRNDE sponges miR-384 to promote proliferation and metastasis of pancreatic cancer cells through upregulating IRS1. Cell Prolif. 2017;50(6) Epub ahead of print.

73. Aken BL, Achuthan P, Akanni W, et al. Ensembl 2017. Nucleic Acids Res. 2017;45(D1):D635-D642. 
OncoTargets and Therapy

\section{Publish your work in this journal}

OncoTargets and Therapy is an international, peer-reviewed, open access journal focusing on the pathological basis of all cancers, potential targets for therapy and treatment protocols employed to improve the management of cancer patients. The journal also focuses on the impact of management programs and new therapeutic agents and protocols on

patient perspectives such as quality of life, adherence and satisfaction. The manuscript management system is completely online and includes a very quick and fair peer-review system, which is all easy to use. Visit http://www.dovepress.com/testimonials.php to read real quotes from published authors.

Submit your manuscript here: http://www.dovepress.com/oncotargets-and-therapy-journal 